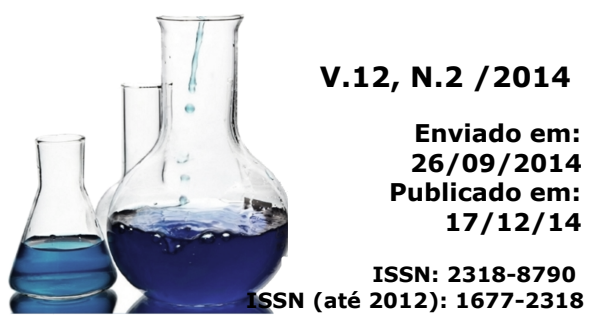

\title{
Realidade aumentada no ensino de vias metabólicas
}

Augmented reality approach for metabolic pathways teaching

\author{
Juan Carlos Vega Garzón, Márcio Luiz Magrini, Caetano da Costa, Eduardo Galembeck \\ Departamento de Bioquímica e Biologia Tecidual - Instituto de Biologia - UNICAMP \\ Agência de fomento: CNPq, PNPD CAPES e Colciencias/Colombia \\ *e-mail: eg@unicamp.br
}

\section{Resumo}

Um quebra-cabeças da glicólise em papel tem sido utilizado como estratégia para o ensino de metabolismo. Essa forma de jogo em papel demanda um número grande de instrutores e limita o acompanhamento das dificuldades apresentadas pelos alunos. A tecnologia denominada Realidade Aumentada (RA) foi empregada para favorecer o uso da estratégia de montagem de vias metabólicas em classes numerosas e armazena dados de utilização, que fornecem feedback para alunos e professores. Cartas com estruturas moleculares são lidas por um aplicativo instalado em dispositivos móveis que exibem informações como a estrutura 3D das moléculas, pistas para a montagem da sequência correta da via metabólica e resultados da progressão na atividade. A utilização de RA possibilitou maior autonomia para os estudantes resolverem os exercícios propostos e fornece dados de avaliação de desempenho que permite compreender, e posteriormente sanar, as dificuldades apresentadas pelos alunos.

Palavras-chave: Vias metabólicas, Realidade aumentada, Aprendizagem ativa.

\begin{abstract}
A glycolysis paper puzzle has been used as strategy to teach metabolic pathways, but this kind of game demands a higher number of instructors and limits the follow up of the students' difficulties. A technology called Augmented Reality (AR) was applied to enable the puzzle usage in large audiences, and to provide feedback to students and instructors. Drafted as flashcards readable by an app installed in tablets, it conveys information as molecules 3D-structure, clues for correct assembling of the metabolic pathway and results of student progression in the activity. Such technological improvement brought more autonomy to students for solving proposed exercises and an embedded performance data collection system helpful to understand, and after to unravel students' difficulties.
\end{abstract}

Keywords: Metabolic pathways, Augmented reality, Active learning 


\section{Ficha da atividade desenvolvida}

Título: Realidade aumentada no ensino de vias metabólicas.

Público alvo: Estudantes universitários envolvidos em disciplinas de Bioquímica.

Disciplinas relacionadas: Bioquímica metabólica.

Objetivos educacionais: Auxiliar a aprendizagem de temas complexos.

Justificativa de uso: Elevado grau de abstração e de complexidade envolvidos no estudo e aprendizagem das vias metabólicas.

Conteúdos trabalhados: Glicólise e Ciclo de Krebs.

\section{Link de acesso do aplicativo:}

Para dispositivos Android:

https://play.google.com/store/apps/details?id=|te.ib.unicamp.br.armet

Para dispositivos iOS:

https://itunes.apple.com/us/app/armet/id874543777?mt=8 


\section{Introdução}

A educação superior passa por uma mudança de paradigma em relação ao ensino e à aprendizagem; de um ensino centrado no professor (metodologia tradicional) para processos de aprendizagem focados nos estudantes, caracterizando as chamadas metodologias ativas de ensino-aprendizagem [1-3].

Desde o advento da Internet em meados da década de 1990, estabeleceu-se uma geração de aprendizes visuais, que cresceram em meio à interatividade inerente a ambientes digitais e dispositivos eletrônicos com tecnologia cada vez mais sofisticada. Apresentam, em geral, aversão a leituras mais extensas e profundas, além de um baixíssimo limiar para assistir a aulas meramente expositivas [4,5].

O ensino e a aprendizagem das disciplinas de Bioquímica são desafiadores pela própria natureza desse conhecimento. Por exemplo, a Bioquímica possui uma terminologia ampla e específica (nomes, fórmulas e estruturas químicas), exige um alto grau de abstração [6,7] e um conhecimento básico de Química. Esta é uma área complexa da qual os alunos trazem, em geral, uma série de conceitos incompletos ou equivocados $[8,9]$.

Naquele contexto de múltiplos estímulos virtuais, é imprescindível o papel do professor, a fim de planejar e efetuar a transposição didática do conteúdo bioquímico, com especial atenção ao método a ser empregado. Atualmente, o uso de metodologias ativas envolvendo a utilização de ambientes virtuais [10-12] e estratégias de resolução de problemas $[13,14]$ tornam o ensino mais participativo e a aprendizagem mais significativa.

Nos últimos anos, tem havido uma tendência ao uso de estratégias visuais associadas a ambientes virtuais de aprendizagem (AVAs) em cursos universitários [7,1517]. Nesse contexto, dispositivos móveis como tablets e smartphones são parte de uma nova realidade em vários campos de atuação profissional. Tais novas tecnologias têm proporcionado metodologias eficientes na obtenção de resultados positivos, como os softwares educativos, que têm a grande capacidade de despertar a curiosidade e o interesse do estudante sobre conteúdos abordados em aplicativos. Assim, professores e estudantes encontram nesses softwares uma ferramenta poderosa para conferir uma nova dimensão ao conhecimento, facilitando a aprendizagem de temas complexos.

Na disciplina de Metabolismo, oferecida ao curso de Ciências Biológicas em uma universidade pública, uma estratégia de ensino foi implementada no ano de 2014: o 
aplicativo ARMET (Augmented Reality METabolic pathways).

O objetivo deste trabalho é apresentar o ARMET e sua aplicação em sala de aula, buscando proporcionar novas formas visuais e interativas que estimulem a aprendizagem das vias metabólicas. Trata-se de uma ferramenta tecnológica cuja linha de pesquisa vem se destacando na literatura recente, denominada "Realidade Aumentada" [18-21].

\section{Procedimentos e recursos}

A dificuldade no ensino-aprendizagem das vias metabólicas, decorrente do novo perfil de aprendiz e dos elementos desafiadores apontados, pode ser contornada por meio de formas alternativas que facilitem a abstração necessária à compreensão do tema. Durante muitos anos, na universidade estudada, tem sido utilizado um quebra-cabeças da glicólise em papel (Figura 1), com o apoio de instrutores em sala de aula. Contudo, notouse que o sucesso da atividade depende do atendimento adequado a todos os estudantes, o que não ocorre devido ao número insuficiente de instrutores. A fim de aplicar a atividade em classes mais numerosas, foi incluído na atividade de montagem do quebra-cabeças de vias metabólicas um aplicativo que envolve Realidade Aumentada [22].

O ARMET consiste em um software educacional que pode ser usado em smartphones e tablets com câmera de vídeo. Esse aplicativo pode ser usado nas principais plataformas disponíveis no mercado, Google/Android e Apple/iOS, aproveitando ao máximo o potencial que tais sistemas apresentam, como a interatividade com o usuário através de toques na tela. Por meio da impressão de cartas em papel comum, estas tomam a forma de moléculas virtuais pelo reconhecimento de padrões presentes nas cartas (flashcards virtuais) via câmera de vídeo do dispositivo móvel. Assim, o usuário tem liberdade de movimento para apontar a carta desejada, podendo criar um ambiente virtual diferente a cada interação.

O ARMET permite a visualização de modelo tridimensional das moléculas envolvidas e a checagem dos resultados conforme o estudante progride na atividade. Por meio da leitura das cartas virtuais, os tablets disponibilizam em suas telas informações como a estrutura 3D das moléculas e pistas para a montagem do quebra-cabeças, cujo objetivo é organizar a sequência correta da via metabólica. Além da glicólise, existem flashcards que permitem o estudo do ciclo de Krebs, e estão em desenvolvimento flashcards para as vias de síntese de hormônios esteroides. 


\section{Desenvolvimento da atividade}

Nos últimos anos, a introdução ao ensino de vias metabólicas em sala de aula era realizada por meio de cartas impressas em papel, em que os estudantes deveriam completar a via metabólica sugerida com as cartas contendo apenas a fórmula estrutural da molécula e seu nome, ou apenas seu nome. Apresenta-se na Figura 1 a sequência final completa do quebra-cabeças da via glicolítica em papel.

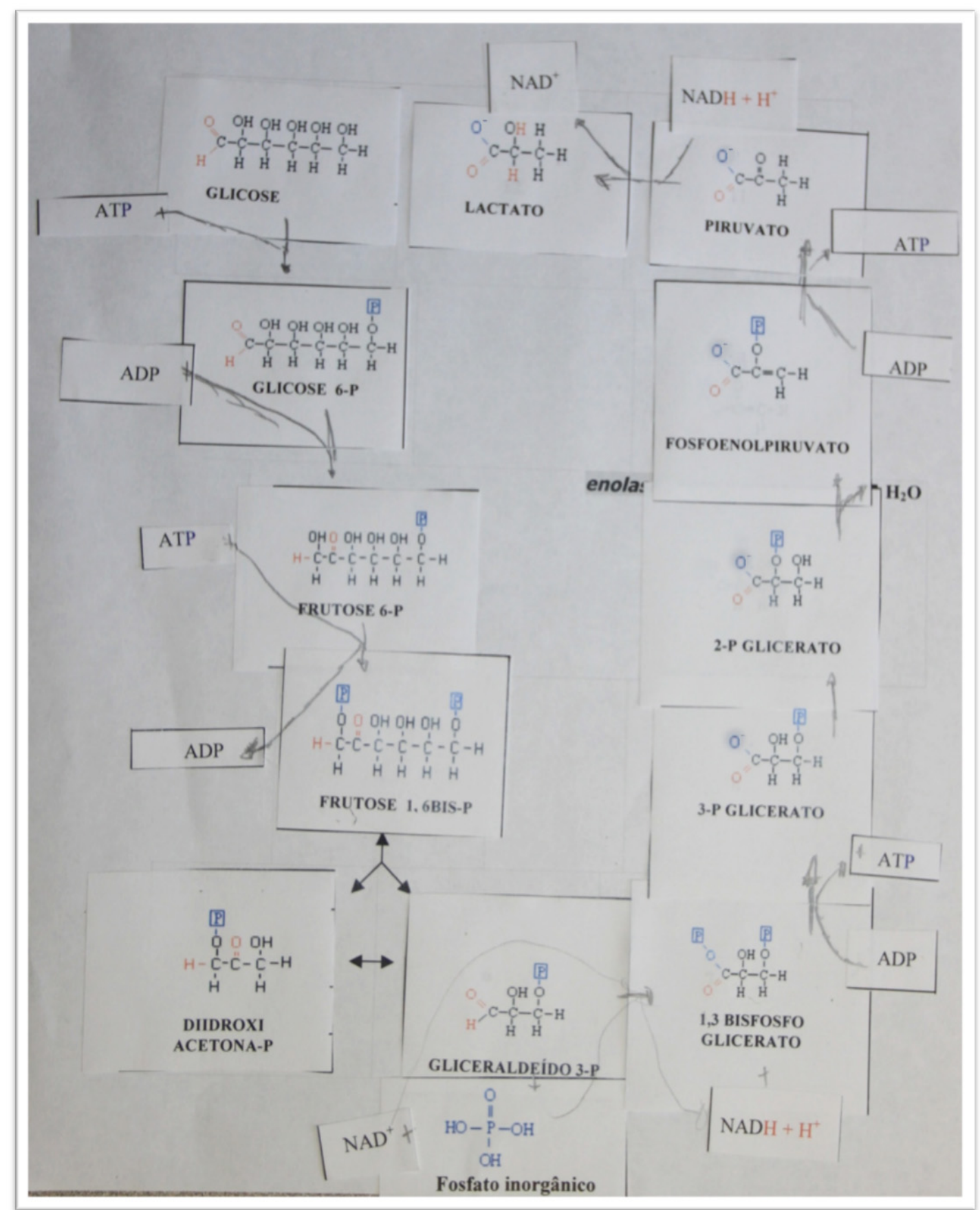

Figura 1. Cartas em papel mostrando o resultado final da montagem do quebra-cabeças (via glicolítica). 
Com o uso do ARMET, é possível aliar o conteúdo das cartas ao poder de geração de modelos tridimensionais na tela do dispositivo móvel, conferindo maior dinamismo ao ensino das vias metabólicas. Apresenta-se na Figura 2 um exemplo da tela do dispositivo com as moléculas em 3D e as informações associadas. Na prática em sala de aula, prevista para durar cerca de duas horas, os estudantes são inicialmente divididos em pequenos grupos (cerca de cinco integrantes cada) e cada grupo recebe um tabuleiro do jogo, um conjunto de cartas com a estrutura química de todos os componentes da via e um dispositivo móvel.

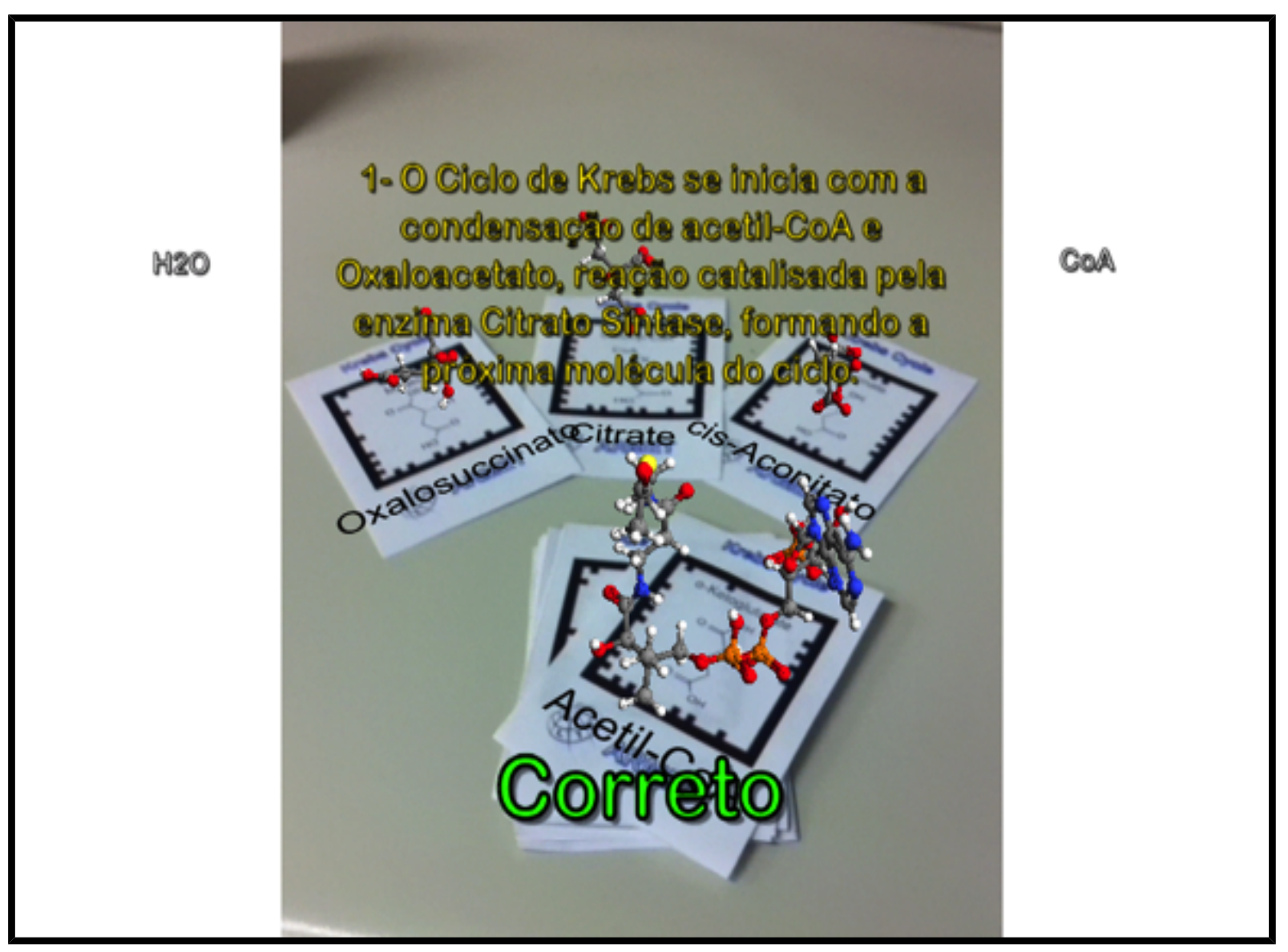

Figura 2. Tela de um tablet mostrando as moléculas em 3D com uma pista relativa à via em estudo (no caso, o ciclo de Krebs).

Após duas aulas teóricas de introdução ao metabolismo e seus princípios de regulação, os estudantes têm a primeira aula sobre degradação de carboidratos (glicólise). A aula consiste em uma atividade interativa em que cada estudante recebe um estudo dirigido com orientações sobre como utilizar o dispositivo móvel para montar uma sequência lógica da via e pistas para orientação (Figura 3). A atividade deve ser realizada sem consulta ao livro-texto ou outra referência; as pistas sobre a glicólise alertam sobre o 
envolvimento de coenzimas em certas reações e o gasto/produção de ATP em outras. Mesmo com a familiaridade no manuseio dos equipamentos, é natural que surjam questões; assim, os estudantes têm à sua disposição, além do professor, em média dois instrutores para fornecer orientações e tirar dúvidas.
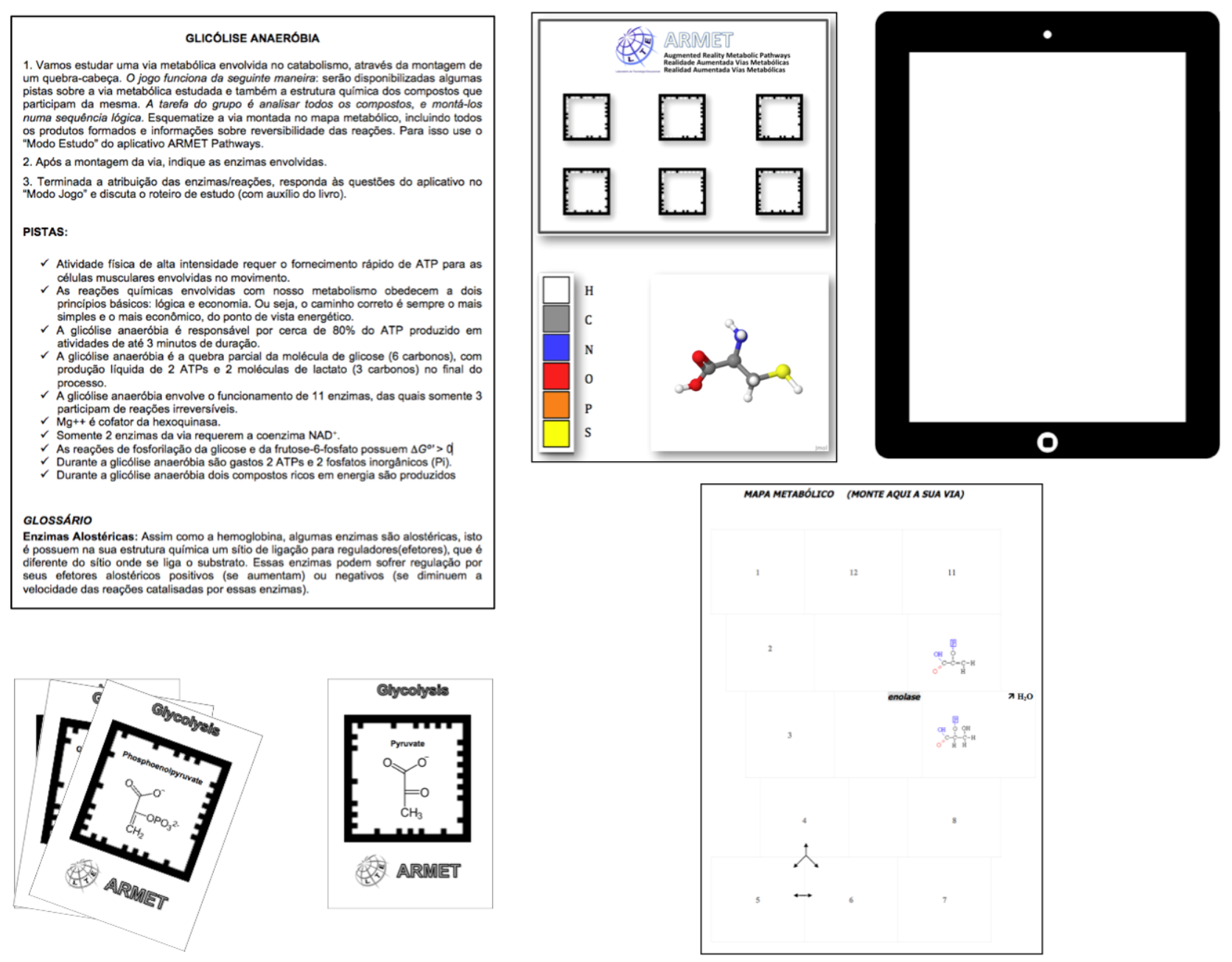

Figura 3. Elementos que cada grupo recebe para realizar a atividade. Cada grupo recebe um dispositivo com o aplicativo instalado (ARMET) (canto superior direito), além das flashcards virtuais com a estrutura química de todos os componentes da via (canto inferior esquerdo) e um tabuleiro (acima, ao centro). Cada estudante recebe um estudo dirigido (canto superior esquerdo) com orientações sobre como utilizar o dispositivo móvel para montar a via no mapa metabólico impresso (canto inferior direito).

O ARMET permite a navegação em dois modos: o modo Jogo, no qual o estudante deve completar a via escolhida com algum elemento faltante, uma molécula; e o modo Estudo, que fornece uma dica que remete para a primeira molécula da via (Figura 4). Dentre todas as cartas disponíveis, o estudante deverá escolher a carta correta para que ele possa receber a dica para a próxima molécula, e assim subsequentemente, até que a via seja completada. Apresenta-se na Figura 5 uma dica que é exibida para o aluno 
quando ele escolhe corretamente a glicose como sendo a primeira molécula da glicólise. Neste caso, aparece na tela um pequeno texto (contendo informações sobre o tipo de reação catalisada e a enzima responsável) como uma dica para que o estudante identifique a próxima molécula da via metabólica.

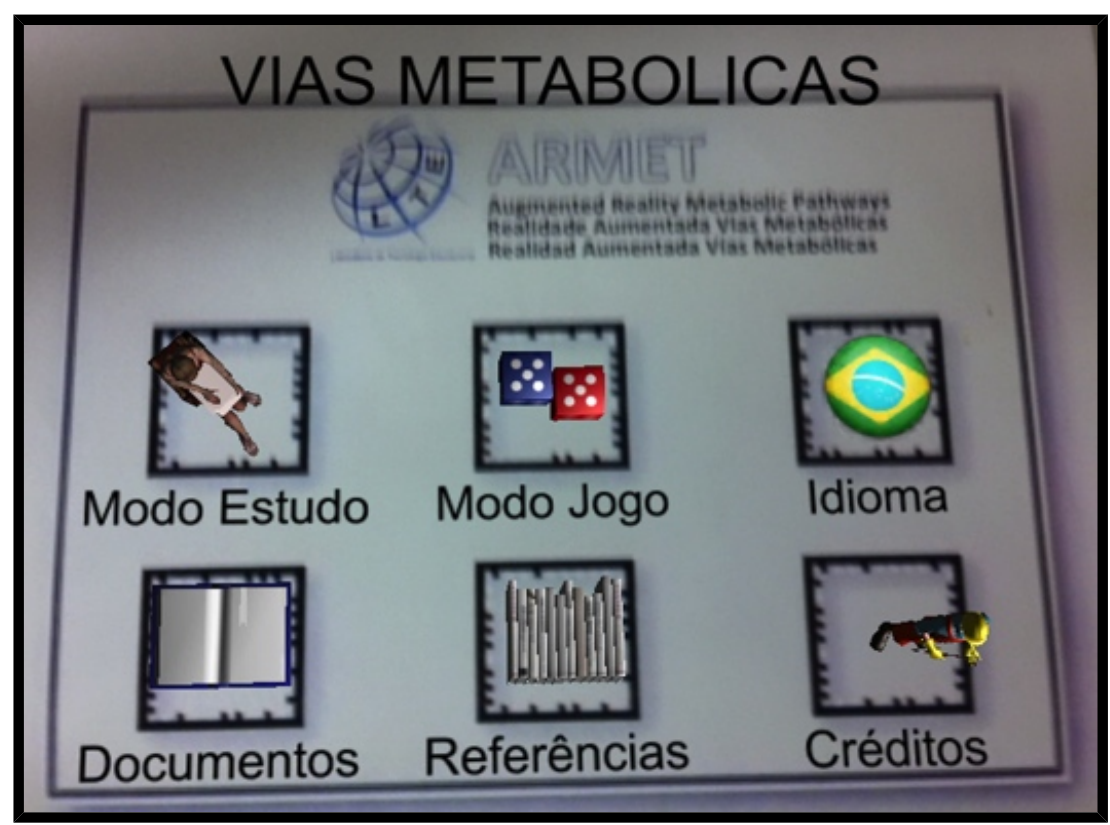

Figura 4. O ARMET permite a navegação em modos: o modo Jogo, no qual o estudante deve completar a via escolhida com algum elemento faltante, e o modo Estudo, que fornece uma dica que remete para a primeira molécula da via.

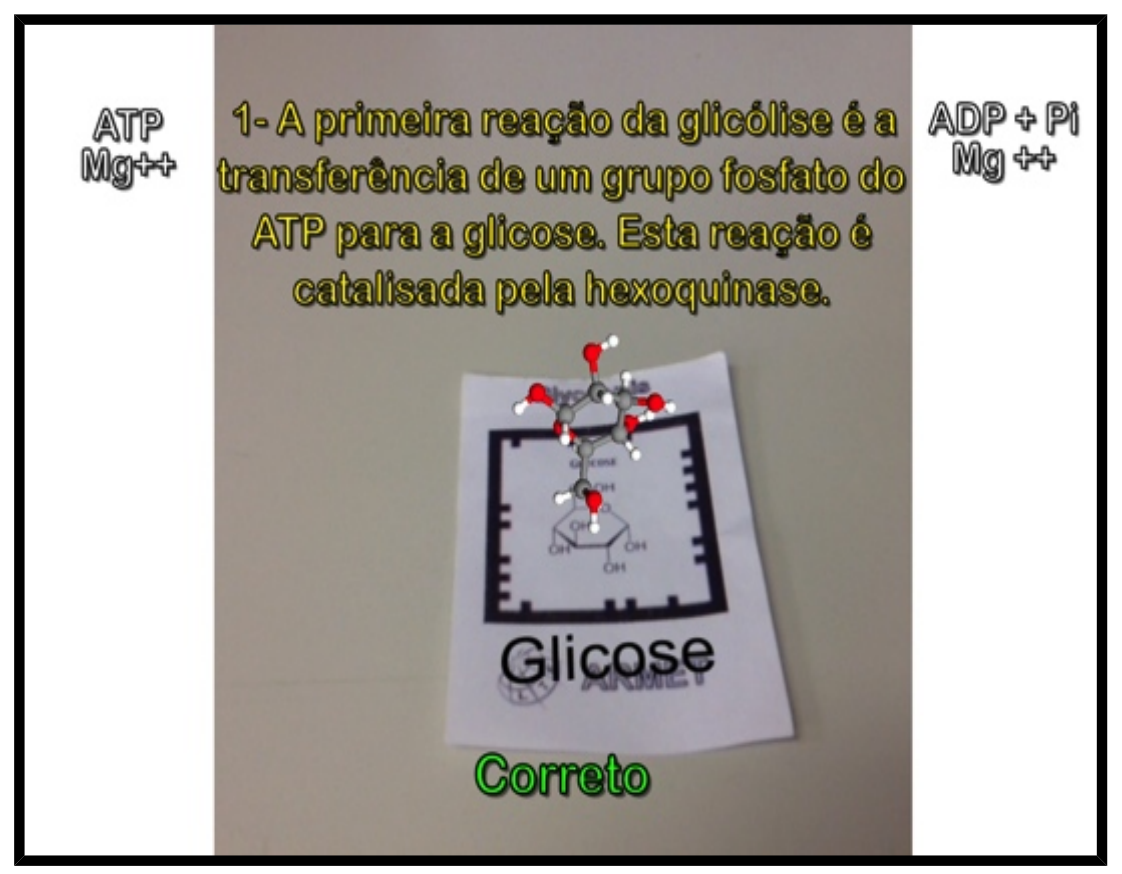

Figura 5. Uma dica que é exibida para o aluno quando ele escolhe corretamente a glicose como sendo a primeira molécula da glicólise. Neste caso, aparece na tela um texto (contendo informações sobre o tipo de reação catalisada e a enzima responsável) com uma dica para que o estudante identifique a próxima molécula da via metabólica. 
Em seguida, os alunos jogarão o modo Jogo e poderão fazer várias tentativas para encontrar a resposta correta para as perguntas randomizadas (10 questões são sorteadas por jogo) na tela do dispositivo móvel (Tabela 1).

Tabela 1. Questões do modo Jogo do ARMET.

\begin{tabular}{|c|l|}
\hline Número & \multicolumn{1}{c|}{ Questões } \\
\hline 1 & A glicólise é responsável por oxidar qual molécula? \\
\hline 2 & Qual é o produto final da glicólise? \\
\hline 3 & $\begin{array}{l}\text { Qual é a molécula energética mais abundante e importante, chamada de "moeda de energia", } \\
\text { de um ser vivo? }\end{array}$ \\
\hline 4 & Qual é o destino metabólico da di-hidroxiacetona fosfato na via glicolítica? \\
\hline 5 & Qual molécula é clivada em duas trioses-fosfato? \\
\hline 6 & Qual molécula tem ativação em reações redox? \\
\hline 7 & Qual molécula é obtida a partir da isomerização da glicose 6-fosfato? \\
\hline 9 & Qual a última molécula da via glicolítica que tem um grupo fosfato removido? \\
\hline 10 & Qual é o substrato da primeira reação de fosforilação da glicólise? \\
\hline 11 & Qual é o substrato da primeira reação de isomerização da via glicolítica? \\
\hline 12 & Qual é o substrato da segunda reação de fosforilação da via glicolítica? \\
\hline 13 & Qual é o produto da segunda reação de isomerização da via glicolítica? \\
\hline 14 & Qual é o substrato da mutase na via glicolítica? \\
\hline 15 & Qual é o produto da mutase na via glicolítica? \\
\hline 16 & Qual é o produto da segunda reação de fosforilação da glicólise? \\
\hline 17 & $\begin{array}{l}\text { Na obtenção de qual molécula o Mg }{ }^{2+} \text { aparece participando como cofator pela primeira vez, } \\
\text { durante a glicólise? }\end{array}$ \\
\hline 18 & Qual é o produto da reação em que é produzido o NADH? \\
\hline 19 & Qual é o substrato da segunda reação da via glicolítica que resulta na formação de ATP? \\
\hline 20 & Qual é o substrato da primeira reação da via glicolítica que resulta na formação de ATP? \\
\hline
\end{tabular}

Tanto no modo Estudo como no modo Jogo, o aplicativo também é capaz de coletar e armazenar os dados de uso em uma base de dados. A base de dados escolhida foi a MySQL, já que o software estatístico R apresenta um pacote (RMySQL) que permite fazer uma conexão com a base de dados para realizar as análises estatísticas e exibir os resultados ao professor em tempo real. Tais dados referem-se ao número de tentativas realizadas para se chegar às respostas corretas e opções incorretas selecionadas. No modo Jogo é ainda armazenada a pontuação do estudante com base no grau de sucesso para atingir a resposta certa com menor número de tentativas. Portanto, durante a montagem do quebra-cabeças com apoio virtual, os estudantes podem avaliar seu desempenho por meio da checagem de suas notas.

As ações do aluno ao utilizar o aplicativo são armazenadas na referida base de dados (MySQL) e tratados usando o software estatístico $R$ para fazer testes de estatística descritiva (média, desvio padrão e percentual de erros corrigido e total), com o objetivo de 
avaliar a dificuldade/facilidade das questões [24].

Todo esse procedimento também é aplicado ao estudo do ciclo de Krebs, com algumas diferenças: no modo de Estudo, basta o estudante selecionar a via (ele terá à disposição duas delas, glicólise ou ciclo de Krebs) e dar seguimento ao estudo; no caso do ciclo de Krebs, para a realização da atividade proposta é permitida a consulta ao livrotexto [23].

\section{Resultados}

A primeira etapa do uso da estratégia de ensino de vias metabólicas, com uso de Realidade Aumentada, envolve a montagem da via metabólica e registro da mesma em uma folha de papel. Após a realização do modo Estudo, os alunos usam o modo Jogo, quando devem responder uma série de perguntas.

O aplicativo foi testado por duas turmas do curso de Ciências Biológicas, sendo 45 do diurno e 45 do noturno. Ao todo, os 90 alunos formaram 12 grupos; no modo Jogo da glicólise, houve registro de respostas às perguntas em 629 vezes, sendo registradas 1.056 tentativas quando incluídas as perguntas visualizadas mas não respondidas. Das perguntas respondidas, $440(69,95 \%)$ respostas estavam corretas na primeira tentativa, $130(20,67 \%)$ requereram de 1 a 5 tentativas e $59(9,38 \%)$ necessitaram de mais de 5 tentativas para encontrar a resposta correta. Apresenta-se na Tabela 2 o número de erros por questão e o percentual de erros por questão.

Na segunda coluna pode ser visto que existe uma grande diferença no número de vezes que cada questão foi mostrada na tela do dispositivo. Essa diferença pode ser explicada porque inicialmente, no estudo piloto, o aplicativo tinha apenas 10 perguntas e depois foram incluídas mais 10 perguntas. O banco de perguntas atual tem 20 questões. Cada vez que uma nova seção começa, o aplicativo mostra aleatoriamente só 10 perguntas no modo Jogo.

A terceira coluna mostra o percentual de erros corrigido pelo número de vezes que a questão foi mostrada na tela do dispositivo. Este dado será utilizado para avaliar quais questões foram mais fáceis e mais difíceis e buscar uma possível explicação para sua dificuldade/facilidade. 
Metodologias inovadoras: Realidade aumentada no ensino de vias metabólicas

Tabela 2. Número e percentual de erros por questão.

\begin{tabular}{|c|c|c|c|c|}
\hline Questão & $\begin{array}{c}\text { Número de vezes } \\
\text { que a questão foi } \\
\text { mostrada na tela } \\
\text { do dispositivo }\end{array}$ & $\begin{array}{c}\text { Número de } \\
\text { erros por } \\
\text { questão }\end{array}$ & $\begin{array}{c}\text { Percentual de } \\
\text { erros corrigido } \\
\text { pelo número de } \\
\text { vezes }\end{array}$ & $\begin{array}{c}\text { Percentual de } \\
\text { erros total por } \\
\text { questão }\end{array}$ \\
\hline 1 & 55 & 10 & 18,18 & 1,59 \\
\hline 2 & 67 & 17 & 25,37 & 2,70 \\
\hline 3 & 57 & 13 & 22,81 & 2,07 \\
\hline 4 & 62 & 15 & 24,19 & 2,38 \\
\hline 5 & 35 & 8 & 22,86 & 1,27 \\
\hline 6 & 54 & 15 & 27,78 & 2,38 \\
\hline 7 & 50 & 10 & 20,00 & 1,59 \\
\hline 8 & 39 & 10 & 25,64 & 1,59 \\
\hline 9 & 26 & 13 & 50,00 & 2,07 \\
\hline 10 & 32 & 11 & 34,38 & 1,75 \\
\hline 11 & 24 & 6 & 25,00 & 0,95 \\
\hline 12 & 31 & 10 & 32,26 & 1,59 \\
\hline 13 & 8 & 4 & 50,00 & 0,64 \\
\hline 14 & 12 & 7 & 58,33 & 1,11 \\
\hline 15 & 12 & 5 & 41,67 & 0,79 \\
\hline 16 & 21 & 8 & 38,10 & 1,27 \\
\hline 17 & 7 & 4 & 57,14 & 0,64 \\
\hline 18 & 10 & 6 & 60,00 & 0,95 \\
\hline 19 & 15 & 9 & 60,00 & 1,43 \\
\hline 20 & 12 & 8 & 66,67 & 1,27 \\
\hline
\end{tabular}

A questão com o maior percentual de acertos foi: "A glicólise é responsável por oxidar qual molécula?". Uma possível explicação é que o próprio nome da via metabólica fornece uma dica para responder à pergunta.

A questão com o menor percentual de acertos foi: "Qual é o substrato da primeira reação da via glicolítica que resulta na formação de ATP?". Uma possível explicação é que a pergunta envolve muitos conceitos específicos da bioquímica e com um alto grau de abstração, por exemplo, "substrato", "reação", "ATP”, tornando-se necessário que o aluno compreenda muito bem esses conceitos para colocá-los em um contexto apropriado e poder resolver a questão.

A Figura 6 apresenta o resumo da estatística descritiva e o boxplot da distribuição dos dados, número de erros total por questão e percentual de erros total por questão. Os resultados mostram que o percentual máximo de erro é menor do que $3 \%(2,70)$ e que o desvio padrão é também pequeno. Os resultados podem ser interpretados como uma alta porcentagem de sucesso dos estudantes na resolução dos exercícios. 
Erros

\begin{tabular}{|c|c|c|}
\hline Min. & $\begin{array}{c}\text { Número de } \\
\text { erros por } \\
\text { questão }\end{array}$ & $\begin{array}{c}\text { Percentual de } \\
\text { erros por } \\
\text { questão }\end{array}$ \\
\hline 1st Qu. & 4,00 & 0,64 \\
\hline Median & 9,75 & 1,07 \\
\hline Mean & 9,45 & 1,51 \\
\hline 3rd Qu & 11,50 & 1,50 \\
\hline Max. & 17,00 & 1,83 \\
\hline sd & 3,72 & 2,70 \\
\hline
\end{tabular}

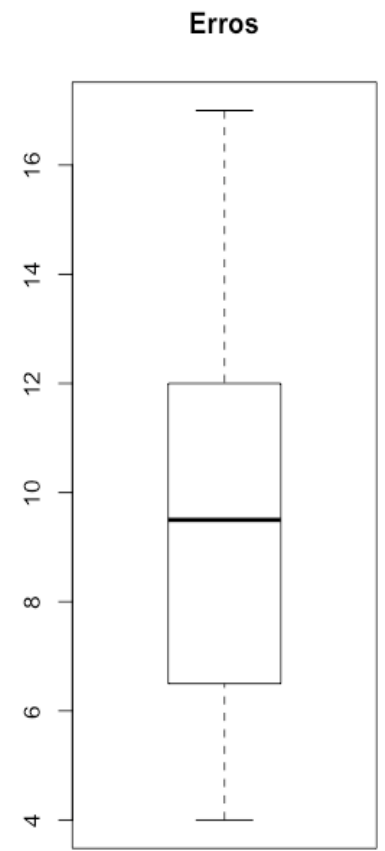

Percentual

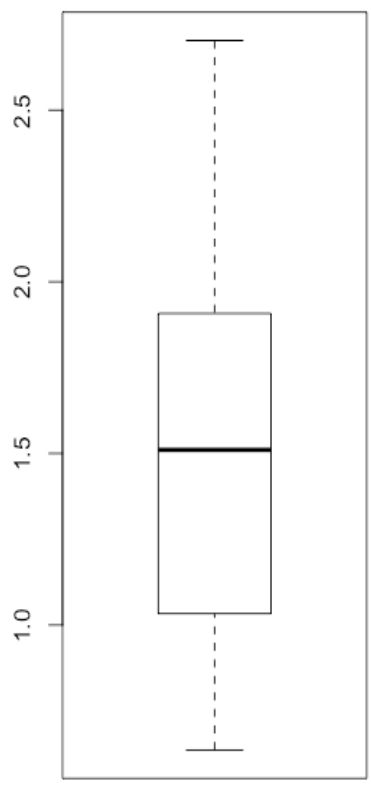

Figura 6. Resumo da estatística descritiva e o boxplot da distribuição dos dados do número de erros total por questão e percentual total de erros por questão.

Em suma, os resultados obtidos mostraram uma alta porcentagem de sucesso (70\%) para resolver os exercícios propostos. Também foi observado que os alunos realizaram um debate acadêmico com os colegas, estimulando a aprendizagem colaborativa. Para se chegar aos resultados obtidos, foram realizados inúmeros testes de usabilidade e jogabilidade, incorporando mudanças de acordo com a necessidade. Encontra-se em fase de teste a implementação de melhorias nas moléculas, como a adição de rotação para que o usuário do aplicativo tenha a possibilidade de observá-las por todos os seus ângulos. Além disso, estuda-se a adição de algumas animações importantes, como a transição detalhada das moléculas da via, a fim de que o aluno entenda quais elementos são essenciais e o papel de cada um deles em cada etapa da via.

Ainda, com base nos resultados coletados no modo Jogo, está sendo implementado um sistema de ranking online. O objetivo é que professor possa usar o aplicativo em sala de aula a fim de avaliar seus alunos através do conhecimento apresentado na resolução das diferentes vias metabólicas. 


\section{Conclusões}

Os aplicativos que utilizam a Realidade Aumentada aliam a capacidade gráfica, a mobilidade e a máxima interatividade daqueles aparelhos para proporcionar ao usuário uma experiência visual única. $\mathrm{O}$ uso de tais dispositivos na educação pode propiciar a distribuição de conteúdo multimídia com alto grau de interatividade, possibilitando o desenvolvimento de atividades educativas individuais ou colaborativas. Ainda, é possível avaliar o desempenho em tempo real, de modo que professores e instrutores possam identificar as dificuldades de aprendizagem dos alunos de forma bastante eficiente, podendo interferir precocemente nessas situações.

Tal melhoria tecnológica permite a aplicação para turmas com maior número de estudantes, com poucos instrutores, uma vez que o aplicativo oferece acesso a diversas informações e permite a discussão com os colegas. Assim, o quebra-cabeças via flashcards se configura como um meio de interação entre os estudantes e como um apoio para atuarem com maior autonomia na resolução dos exercícios propostos.

Do ponto de vista da aprendizagem, os resultados deste estudo piloto mostraram que a Realidade Aumentada tem o potencial de ajudar os estudantes a adquirir as ferramentas intelectuais e de contribuir para desenvolver as habilidades necessárias para a compreensão dos fundamentos bioquímicos. Por exemplo, a compreensão do que é uma via metabólica, quais são seus princípios básicos, seus conceitos essenciais (substrato, produto, tipos de reações e enzimas) e seus conceitos necessários (fontes de energia, cinética da reação, estequiometria, cofatores e coenzimas). Na prática, aquele potencial é aproveitado não como um conjunto de exercícios de memorização, mas como um processo de aprendizagem significativa.

\section{Referências}

[1] Mitre SM, Siqueira-Batista R, Girardi-de-Mendonça JM, Morais-Pinto NM, Meirelles CAB, Pinto-Porto $C$, et al. Metodologias ativas de ensino-aprendizagem na formação profissional em saúde: debates atuais. Cien Saude Colet. 2008; 13 Suppl 2: 2133-44.

[2] Berbel NAN. As metodologias ativas e a promoção da autonomia de estudantes. Semin Ciênc Soc Hum. 2011; 32(1): 25-40.

[3] Gardner J, Belland JR. A conceptual framework for organizing active learning experiences in biology instruction. J Sci Educ Technol. 2012; 21(4): 465-75. 
[4] Thompson P. The digital natives as learners: technology use patterns and approaches to learning. Comput Educ. 2013 Jul; 65: 12-33.

[5] Alan R. The digital natives are restless: inspiring a new generation of learners. Sch Bus Aff. 2012; 78(1): 8-10.

[6] Schönborn KJ, Anderson TR. Bridging the educational research-teaching practice gap: foundations for assessing and developing biochemistry students' visual literacy. Biochem Mol Biol Educ. 2010; 38(5): 347-54.

[7] Santos VJSV, Oliveira EA, Vega Garzón JC, Galembeck E. Desenvolvimento e avaliação de uma ferramenta para diagnóstico de literacia visual, contextualizada no ensino de metabolismo. Rev Ensino Bioquím. 2013; 11(1): 54-88.

[8] Barke H, Hazari A, Yitbarek S. Misconceptions in chemistry: Addressing perceptions in chemical education. Berlin: Springer-Verlag; 2009.

[9] Villafañe SM, Bailey CP, Loertscher J, Minderhout V, Lewis JE. Development and analysis of an instrument to assess student understanding of foundational concepts before biochemistry coursework. Biochem Mol Biol Educ. 2011; 39(2): 102-9.

[10] Heidrich DN, Angotti JAP. Implantação e avaliação de ensino semipresencial em disciplinas de bioquímica utilizando ambiente virtual de aprendizagem. Rev Bras Ens Bioq Biol Mol. 2010; 8(1): 45-58.

[11] Barrette- $\mathrm{Ng} \mathrm{IH}$. The pH ruler: a Java applet for developing interactive exercises on acids and bases. Biochem Mol Biol Educ. 2011; 39(4): 332-38.

[12] Taylor A. Teaching biochemistry and molecular biology in 3D: the new next generation science standards. Biochem Mol Biol Educ. 2013; 41(5): 348-50.

[13] Hartfield PJ. Reinforcing constructivist teaching in advanced level biochemistry through the introduction of case-based learning activities. J Learn Des. 2010; 3(3): 20-31.

[14] Van Merrienboer JJG. Perspectives on problem solving and instruction. Comput Educ. 2013 May; 64: 153-60.

[15] Mueller D, Strohmeier S. Design characteristics of virtual learning environments: state of research. Comput Educ. 2011; 57(4): 2505-16.

[16] Wardenski RF, Espindola MB, Struchiner M, Giannella TR. Blended learning in biochemistry education: analysis of medical students' perceptions. Biochem. Mol. Biol. Educ. 2012; 40(4): 222-8.

[17] Teri S, Acai A, Griffith D, Mahmoud Q, Ma DWL, Newton G. Student use and pedagogical impact of a mobile learning application. Biochem Mol Biol Educ. 2014; 42(2): 121-35.

[18] Wu H, Lee SW, Chang H, Liang J. Current status, opportunities and challenges of augmented reality in education. Comput Educ. 2013 Mar; 62: 41-9. 
[19] Annetta L, Burton EP, Frazier W, Cheng R, Chmiel M. Augmented reality games: using technology on a budget. Sci Scope. 2012; 36(3): 54-60.

[20] Munnerley D, Bacon M, Wilson A, Steele J, Hedberg J, Fitzgerald R. Confronting an augmented reality. Res Learn Technol. 2012; 20 Suppl: 39-48.

[21] Lee $\mathrm{K}$. The future of learning and training in augmented reality. InSight $\mathrm{J}$ Sch Teach. 2012; 7: 31-42.

[22] Galembeck E, Magrini ML, Vega Garzón JC. Using augmented reality to bring interactivity to metabolism teaching. Anais da XLIII Reunião Anual da Sociedade Brasileira de Bioquímica e Biologia Molecular; 17 a 21 de maio de 2014, Foz do Iguaçu, PR. Sociedade Brasileira de Bioquímica e Biologia Molecular - SBBq, 2014.

[23] Marzzoco A, Torres BB. Bioquímica básica. $3^{\mathrm{a}}$ ed. Rio de Janeiro: Guanabara Koogan; 2007.

[24] Vega Garzón JC, Magrini ML, Galembeck E. Using augmented reality (AR) application to teach the glycolysis. Livro de resumos do II Workshop BFM; 13 a 14 de agosto de 2014, Campinas, SP. p. 22. 